\title{
CENTRAL ELEMENTS AND CANTOR-BERNSTEIN'S THEOREM FOR PSEUDO-EFFECT ALGEBRAS
}

\author{
ANATOLIJ DVUREČENSKIJ
}

(Received 7 May 2001; revised 4 January 2002)

Communicated by B. Davey

\begin{abstract}
Pseudo-effect algebras are partial algebras $(E ;+, 0,1)$ with a partially defined addition + which is not necessary commutative and with two complements, left and right ones. We define central elements of a pseudo-effect algebra and the centre, which in the case of MV-algebras coincides with the set of Boolean elements and in the case of effect algebras with the Riesz decomposition property central elements are only characteristic elements. If $E$ satisfies general comparability, then $E$ is a pseudo MV-algebra. Finally, we apply central elements to obtain a variation of the Cantor-Bernstein theorem for pseudo-effect algebras.
\end{abstract}

2000 Mathematics subject classification: primary 06D35, 03G12, $03 \mathrm{~B} 50$.

Keywords and phrases: Pseudo-effect algebra, effect algebra, central element, general comparability, pseudo MV-algebra, monotone $\sigma$-completeness, Cantor-Bernstein theorem.

\section{Introduction}

Recently two non-commutative generalizations of MV-algebras introduced by Chang [3] have appeared: pseudo MV-algebras of Georgescu and Iorgulescu [13] and generalized MV-algebras of Rachůnek [21] which, in addition, are equivalent. Also a non-commutative version of BL-algebras, pseudo-BL-algebras, have been introduced in [6]. Non-commutative algebras are algebraic non-commutative analogues of noncommutative reasoning. Such reasoning can be met in the everyday life quite often. Many psychological processes are depending on the order of variables. The result is not the same when we first put on our shoes and then socks, or conversely. Today there exists even a programming language [1] based on a non-commutative logic.

Recently in $[9,10]$ we have introduced pseudo-effect algebras as a non-commutative generalization of effect algebras, which play an important role in mathematical foun-

(C) 2003 Australian Mathematical Society $1446-7887 / 03 \$ A 2.00+0.00$ 
dations of quantum mechanics. Effect-algebras were introduced by Foulis and Bennett [11] as an additive counterpart to D-posets introduced by Kôpka and Chovanec [19].

In many cases pseudo-effect algebras are intervals in unital po-groups $(G, u)$ [10], and every pseudo MV-algebra is an interval in a unital $\ell$-group $(G, u)$.

In the present paper we introduce the notion of central elements of pseudo-effect algebras. For effect algebras this was done in [15]. We show that such elements form always a Boolean algebra of $E$. The paper is organized as follows. In Section 2 we define pseudo-effect algebras and their central elements. In the case when the pseudo-effect algebra satisfies a variation of the Riesz decomposition property, we characterize central elements as those elements $e$ satisfying $e \wedge e^{\prime}=0$, Section 3 . In Section 4, we show that any pseudo-effect algebra with general comparability is a pseudo MV-algebra. If $E$ is monotone $\sigma$-complete, then the centre is a Boolean $\sigma$-algebra, Section 5, and finally, a version of the Cantor-Bernstein theorem will be proved.

We recall that Jakubík gave two versions of the Cantor-Bernstein theorem for $\sigma$ complete MV-algebras [16] and for pseudo MV-algebras [17]. Another generalization of the Cantor-Bernstein theorem for $\sigma$-complete MV-algebras is given in [4] and for monotone $\sigma$-complete effect algebras in [18] and for orthomodular lattices in [5].

\section{Central elements of pseudo-effect algebras}

A partial algebra $(E ;+, 0,1)$, where + is a partial binary operation and 0 and 1 are constants, is called a pseudo-effect algebra if, for all $a, b, c \in E$, the following holds:

(i) $a+b$ and $(a+b)+c$ exist if and only if $b+c$ and $a+(b+c)$ exist, and in this case $(a+b)+c=a+(b+c)$;

(ii) there is exactly one $d \in E$ and exactly one $e \in E$ such that $a+d=e+a=1$;

(iii) if $a+b$ exists, there are elements $d, e \in E$ such that $a+b=d+a=b+e$;

(iv) if $1+a$ or $a+1$ exists, then $a=0$.

If we define $a \leq b$ if and only if there exists an element $c \in E$ such that $a+c=b$, then $\leq$ is a partial ordering on $E$ such that $0 \leq a \leq 1$ for any $a \in E$. It is possible to show that $a \leq b$ if and only if $b=a+c=d+a$ for some $c, d \in E$. We write $c=a / b$ and $d=b \backslash a$. Then

$$
(b \backslash a)+a=a+(a / b)=b, \quad \text { and } \quad a=(b \backslash a) / b=b \backslash(a / b) .
$$

If $a \leq b \leq c$, then

$$
\begin{array}{ll}
(c \backslash a) \backslash(b \backslash a)=c \backslash b, & (a / b) /(a / c)=b / c, \\
(c \backslash b) /(c \backslash a)=b \backslash a, & (a / c) \backslash(b / c)=a / b .
\end{array}
$$


Let $E=(E ;+, 0,1)$ be a pseudo-effect algebra. We define $x^{-}:=1 \backslash x$ and $x^{\sim}:=x / 1$ for any $x \in E$. For a given element $e \in E$, we denote by $[0, e]:=$ $\{x \in E: 0 \leq x \leq e\}$. Then $[0, e]$ endowed with + restricted to $[0, e] \times[0, e]$ is a pseudo-effect algebra $[0, e]=([0, e] ;+, 0, e)$. Then, for any $x \in[0, e]$ we have $x^{-\cdot}:=e \backslash x$ and $x^{\sim e}:=x / e$ and $e=x^{-\cdot}+x=x+x^{\sim}$. For basic properties of pseudo-effect algebras see $[9,10]$.

For example if $(G, u)$ is a unital (not necessary Abelian) po-group with strong unit $u$, and $\Gamma(G, u):=[0, u]=\{g \in G: 0 \leq g \leq u\}$, then $(\Gamma(G, u) ;+, 0, u)$ is a pseudo-effect algebra if we restrict the group addition + to $\Gamma(G, u)$.

We recall that a pseudo $M V$-algebra is an algebra $\left(M ; \oplus,^{-}, \sim, 0,1\right)$ of type $(2,1,1$, 0,0 ) such that the following axioms hold for all $x, y, z \in M$ with an additional binary operation $\odot$ defined via $y \odot x=\left(x^{-} \oplus y^{-}\right)^{\sim}$

(A1) $x \oplus(y \oplus z)=(x \oplus y) \oplus z$

(A2) $x \oplus 0=0 \oplus x=x$;

(A3) $x \oplus 1=1 \oplus x=1$;

(A4) $1^{\sim}=0 ; 1^{-}=0$;

(A5) $\left(x^{-} \oplus y^{-}\right)^{\sim}=\left(x^{\sim} \oplus y^{\sim}\right)^{-}$;

(A6) $x \oplus x^{\sim} \odot y=y \oplus y^{\sim} \odot x=x \odot y^{-} \oplus y=y \odot x^{-} \oplus x$;

(A7) $x \odot\left(x^{-} \oplus y\right)=\left(x \oplus y^{\sim}\right) \odot y$;

(A8) $\left(x^{-}\right)^{\sim}=x$.

In [7] it was shown that every pseudo MV-algebra is isomorphic to $\Gamma(G, u)$, where $(G, u)$ is a unital $\ell$-group with strong unit $u$, where $a \oplus b:=(a+b) \wedge u$, $a \odot b=(a-u+b) \vee 0$ and $a^{\sim}=u-a$ and $a^{-}=-a+u$.

If $M$ is a pseudo MV-algebra, then the partial operation $a+b$ is defined if and only if $a \leq b^{-}$, and then $a+b=a \oplus b$, and $(M ;+, 0,1)$ is a pseudo-effect algebra.

DEFINITION 2.1. An element $e$ of a pseudo-effect algebra $E$ is said to be central (or Boolean) if there exists an isomorphism

$$
f_{e}: E \rightarrow[0, e] \times\left[0, e^{\sim}\right]
$$

such that $f_{e}(e)=(e, 0)$ and if $f_{e}(x)=\left(x_{1}, x_{2}\right)$, then $x=x_{1}+x_{2}$ for any $x \in E$.

We denote by $C(E)$ the set of all central elements of $E$, and $C(E)$ is said to be the centre of $E$. We recall that $0,1 \in C(E)$.

PROPOSITION 2.2. Let e be a central element of a pseudo-effect algebra $E$, and $f_{e}$ the corresponding mapping from Definition 2.1. Then

(i) $f_{e}\left(e^{\sim}\right)=\left(0, e^{\sim}\right)$.

(ii) If $x \leq e$, then $f_{e}(x)=(x, 0)$.

(iii) $e \wedge e^{\sim}=0$. 
(iv) If $y \leq e^{\sim}$ then $f_{e}(y)=(0, y)$.

(v) $e^{\sim}=e^{-}$.

(vi) For any $x \in E, x \wedge e \in E$ and $x \wedge e^{\sim} \in E$, and

$$
f_{e}(x)=\left(x \wedge e, x \wedge e^{\sim}\right)
$$

(vii) If $f_{e}(x)=\left(x_{1}, x_{2}\right)$, then $x=x_{1} \vee x_{2}, x_{1} \wedge x_{2}=0$, and $x_{2}+x_{1}=x$.

PROOF. (i) $f_{e}\left(e^{\sim}\right)=f_{e}(e)^{\sim}=(e, 0)^{\sim}=\left(e^{\sim} \cdot, 0^{\sim} \cdot\right)=\left(0, e^{\sim}\right)$.

(ii) Let $f_{e}(x)=\left(x_{1}, x_{2}\right), x \leq e$. Then $\left(x_{1}, x_{2}\right)=f_{e}(x) \leq f_{e}(e)=(e, 0)$, that is, $x_{2}=0$. Hence $x=x_{1}+x_{2}=x_{1}$.

(iii) Let $x \leq e, e^{\sim}$. Then $(x, 0)=f_{e}(x) \leq f_{e}\left(e^{\sim}\right)=\left(0, e^{\sim}\right)$, so that $x=0$. (iv) Let $y \leq e^{\sim}$. Then $f_{e}(y)=\left(y_{1}, y_{2}\right)$, and $y_{1} \leq e, y_{1} \leq y \leq e^{\sim}$ so that by (iii), $y_{1}=0$ and $y=y_{1}+y_{2}=y_{2}$.

(v) $\left(e, e^{\sim}\right)=f_{e}\left(e^{-}+e\right)=f_{e}\left(e^{-}\right)+(e, 0)=\left(e_{1}, e_{2}\right)+(e, 0)=\left(e_{1}+e, e_{2}\right)$ which yields $e=e_{1}+e$ and $e_{2}=e^{\sim}$, that is, $e_{1}=0$ and $f_{e}\left(e^{-}\right)=\left(0, e^{\sim}\right)$ which gives $e^{-}=0+e^{\sim}=e^{\sim}$.

(vi) Let $\pi_{e}$ and $\pi_{e^{\sim}}$ be the projections from $[0, e] \times\left[0, e^{\sim}\right]$ onto $[0, e]$ and $\left[0, e^{\sim}\right]$, respectively. Then $p_{e}:=\pi_{e} \circ f_{e}$ and $p_{e^{\sim}}:=\pi_{e^{\sim}} \circ f_{e}$ are homomorphisms from $E$ into $[0, e]$ and $\left[0, e^{\sim}\right]$, respectively. It is clear that $p_{e}(x) \leq x, e$. If now $y \leq x, e$, then by (ii), $y=p_{e}(y) \leq p_{e}(x)$, so that $e \wedge x=p_{e}(x) \in E$ for any $x \in E$. Similarly, $p_{e^{\sim}} \leq x, e^{\sim}$, and if $z \leq x, e^{\sim}$ then by (iv), $z=p_{e^{\sim}}(z) \leq p_{e^{\sim}}(x)$, that is, $p_{e^{\sim}}(x)=x \wedge e^{\sim}$. Consequently, $f_{e}(x)=\left(x \wedge e, x \wedge e^{\sim}\right), x \in E$.

(vii) It is clear that $x \geq x_{1}, x_{2}$. Let $z \geq x_{1}, x_{2}$. Then $x_{1}=p_{e}\left(x_{1}\right) \leq p_{e}(z)$ and $x_{2}=p_{e^{-}}\left(x_{2}\right) \leq p_{e^{-}}(z)$, so that $x=x_{1}+x_{2} \leq p_{e}(z)+p_{e^{-}}(z)=z$ which proves $x_{1} \vee x_{2}=x$. It is evident that $x_{1} \wedge x_{2}=0$.

By (2) we have $x_{1}=x \wedge e$ and $x_{2}=x \wedge e^{\sim}$. Hence by $(v),(x \wedge e)^{-}=$ $x^{-} \vee e^{-} \geq x \wedge e^{-}=x \wedge e^{\sim}=x_{2}$, which gives $x_{2}+x_{1} \in E$. Then $p_{e}\left(x_{2}+x_{1}\right)=$ $p_{e}\left(x_{2}\right)+p_{e}\left(x_{1}\right)=p_{e}\left(x_{1}\right)=x_{1}$ and $p_{e^{\sim}}\left(x_{2}+x_{1}\right)=p_{e^{\sim}}\left(x_{2}\right)+p_{e^{\sim}}\left(x_{1}\right)=x_{2}$ which proves $x_{2}+x_{1}=x_{1}+x_{2}=x$.

In view of Proposition $2.2(\mathrm{v})$, if $e \in C(E)$, then we will write $e^{\prime}:=e^{-}=e^{\sim}$.

THEOREM 2.3. Let $E$ be a pseudo-effect algebra. If e, $f \in C(E)$, then $e \wedge f \in E$ and $e \wedge f \in C(E)$, and $C(E)=\left(C(E) ; \wedge, \vee,^{\prime}, 0,1\right)$ is a Boolean algebra.

PRoOF. It is evident that $0,1 \in C(E)$. Let now $e \in C(E)$, then $e^{\sim}=e^{-}$and by Proposition 2.2 the mapping $f_{e}(x)=\left(x \wedge e, x \wedge e^{\sim}\right)$ is an isomorphism from $E$ onto $[0, e] \times\left[0, e^{\sim}\right]$. By Proposition 2.2 (vii), we have that the mapping $f_{e^{-}}(x)=(x \wedge$ $\left.e^{-}, x \wedge e\right)$ is an isomorphism from $E$ onto $\left[0, e^{-}\right] \times[0, e]$ such that $f_{e^{-}}\left(e^{-}\right)=\left(e^{-}, 0\right)$ and if $f_{e^{-}}(x)=\left(x_{1}, x_{2}\right)$, then $x=x_{2}+x_{1}=x_{1}+x_{2}$, which proves $e^{\prime} \in C(E)$. 
Assume now $e, f \in C(E)$. Then, for every $x \in E$,

$$
x=x \wedge e+x \wedge e^{\prime}=x \wedge e \wedge f+x \wedge e \wedge f^{\prime}+x \wedge e^{\prime} \wedge f+x \wedge e^{\prime} \wedge f^{\prime}
$$

and in view of $1=e \wedge f+e \wedge f^{\prime}+e^{\prime} \wedge f+e^{\prime} \wedge f^{\prime}$, we have $(e \wedge f)^{\sim}=$ $e \wedge f^{\prime}+e^{\prime} \wedge f+e^{\prime} \wedge f^{\prime}$. On the other hand, $1=f^{\prime}+f=f^{\prime} \wedge e+f^{\prime} \wedge$ $e^{\prime}+f \wedge e^{\prime}+f \wedge e=e \wedge f^{\prime}+e^{\prime} \wedge f+e^{\prime} \wedge f^{\prime}+e \wedge f$, by Proposition 2.6 (ii), so that $(e \wedge f)^{-}=e \wedge f^{\prime}+e^{\prime} \wedge f+e^{\prime} \wedge f^{\prime}=(e \wedge f)^{\sim}$. Hence the mapping $f_{e \wedge f}(x)=\left(x \wedge e \wedge f, x \wedge e \wedge f^{\prime}+x \wedge e^{\prime} \wedge f+x \wedge e^{\prime} \wedge f^{\prime}\right)$ is a well defined mapping from $E$ into $[0, e \wedge f] \times\left[0,(e \wedge f)^{\sim}\right]$ which is injective in view of (3). Moreover, if $f_{e \wedge f}(x)=\left(x_{1}, x_{2}\right)$, then $x_{1}+x_{2}=x$, and $f_{e \wedge f}(e \wedge f)=(e \wedge f, 0)$.

Assume $x_{1} \in[0, e \wedge f], x_{2} \in\left[0,(e \wedge f)^{\sim}\right]$. Then $x_{1} \leq x_{2}^{-}$, so that $x=x_{1}+x_{2} \in E$. Hence $\left(x_{1}+x_{2}\right) \wedge e \wedge f=x_{1} \wedge e \wedge f+x_{2} \wedge e \wedge f=x_{1}+x_{2} \wedge e \wedge f$. On the other side, $x_{2} \leq(e \wedge f)^{\sim}=e \wedge f^{\prime}+e^{\prime} \wedge f+e^{\prime} \wedge f^{\prime}$ so that $x_{2} \wedge e \leq e \wedge f^{\prime}+e^{\prime} \wedge f \wedge e+e^{\prime} \wedge f^{\prime} \wedge e=$ $e \wedge f^{\prime}$ and $x_{2} \wedge e \wedge f \leq e \wedge f^{\prime} \wedge f=0$.

Similarly, $\left(x_{1}+x_{2}\right) \wedge e \wedge f^{\prime}+\left(x_{1}+x_{2}\right) \wedge e^{\prime} \wedge f+\left(x_{1}+x_{2}\right) \wedge e^{\prime} \wedge f^{\prime}=$ $x_{1} \wedge e \wedge f^{\prime}+x_{2} \wedge e \wedge f^{\prime}+x_{1} \wedge e^{\prime} \wedge f+x_{2} \wedge e^{\prime} \wedge f+x_{1} \wedge e^{\prime} \wedge f^{\prime}+x_{2} \wedge e^{\prime} \wedge f^{\prime}$. But $x_{1} \leq e \wedge f$. Then $x_{1} \wedge e \wedge f^{\prime} \leq e \wedge f \wedge e \wedge f^{\prime}=0$ and $x_{1} \wedge e^{\prime} \wedge f \leq e \wedge f \wedge e^{\prime} \wedge f=0$ which proves that $f_{e \wedge f}$ is surjective.

Finally, we show that $f_{\text {e^f }}(x+y)=f_{\text {e^f }}(x)+f_{\text {e^f }}(y)$ whenever $x+y \in E$.

Calculate

$$
\begin{aligned}
(x+y) & \wedge e \wedge f^{\prime}+(x+y) \wedge e^{\prime} \wedge f+(x+y) \wedge e^{\prime} \wedge f^{\prime} \\
= & x \wedge e \wedge f^{\prime}+y \wedge e \wedge f^{\prime}+x \wedge e^{\prime} \wedge f^{\prime}+y \wedge e^{\prime} \wedge f^{\prime} \\
& +x \wedge e^{\prime} \wedge f^{\prime}+y \wedge e^{\prime} \wedge f^{\prime} .
\end{aligned}
$$

Then $x \wedge e^{\prime} \wedge f \leq\left(y \wedge e \wedge f^{\prime}\right)^{-}$so that $x \wedge e^{\prime} \wedge f+y \wedge e \wedge f^{\prime} \in E$. We assert

$$
\begin{aligned}
x \wedge e^{\prime} \wedge f+y \wedge e \wedge f^{\prime} & =\left(x \wedge e^{\prime} \wedge f\right) \vee\left(y \wedge e \wedge f^{\prime}\right) \\
& =y \wedge e \wedge f^{\prime}+x \wedge e^{\prime} \wedge f .
\end{aligned}
$$

Let $z \geq y \wedge e \wedge f^{\prime}, x \wedge e^{\prime} \wedge f$. Then $z \wedge e \wedge f^{\prime} \geq y \wedge e \wedge f^{\prime}$ and $z \wedge e^{\prime} \wedge f \geq x \wedge e^{\prime} \wedge f$. Hence $z \geq z \wedge e \wedge f^{\prime}+z \wedge e^{\prime} \wedge f$ which proves (**). In a similar way we can prove that $y \wedge e^{\prime} \wedge f+x \wedge e^{\prime} \wedge f^{\prime}=x \wedge e^{\prime} \wedge f^{\prime}+y \wedge e^{\prime} \wedge f$. Therefore for $(*)$ we have that it equals to $x \wedge e \wedge f^{\prime}+x \wedge e^{\prime} \wedge f+y \wedge e \wedge f^{\prime}+x \wedge e^{\prime} \wedge f^{\prime}+y \wedge e^{\prime} \wedge f+y \wedge e^{\prime} \wedge f^{\prime}$.

In a similar way, we have that it equals to $x \wedge e \wedge f^{\prime}+x \wedge e^{\prime} \wedge f+x \wedge e^{\prime} \wedge$ $f^{\prime}+y \wedge e \wedge f^{\prime}+y \wedge e^{\prime} \wedge f+y \wedge e^{\prime} \wedge f^{\prime}$. Consequently, $e \wedge f \in C(E)$, and $x \wedge(e \wedge f)^{\sim}=x \wedge e \wedge f^{\prime}+x \wedge e^{\prime} \wedge f+x \wedge e^{\prime} \wedge f^{\prime}$.

Proposition 2.4. Let $x \in E$ and $e \in C(E)$. Then 
(i) $x \wedge e=0$ if and only if $x \leq e^{-}$if and only if $x \leq e^{\sim}$ if and only if $e \leq x^{-}$if and only if $e \leq x^{\sim}$.

(ii) $e+e \in E$ implies $e=0$.

Proof. (i) Let $x \wedge e=0$, then $x=x \wedge e+x \wedge e^{-}=x \wedge e^{-} \leq e^{-}$. Let $x \leq e^{-}$. Then $x \wedge e \leq e^{-} \wedge e=0$. Let $x \wedge e=0$. Then $x \leq e^{\sim}$ and $e \leq x^{-}$. If $e \leq x^{-}$, then $x \leq e^{\sim}=e^{-}$. If $x \wedge e=0$, then $x=x \wedge e+x \wedge e^{-}$that is, $x \leq e^{-}$and $e \leq x^{\sim}$. If $e \leq x^{\sim}$, then $x \leq e^{-}$and $x \wedge e=0$.

(ii) It follows from (i).

PROPOSITION 2.5. Let $e_{1}, \ldots, e_{n} \in C(E), e_{i} \wedge e_{j}=0$ for $i \neq j$, and $e_{1}+\cdots+e_{n}=1$. Then $x=x \wedge e_{1}+\cdots+x \wedge e_{n}$.

PROOF. If $n=1$, then $e_{1}=1$. The general case follows mathematical induction. Let $n \geq 2$. Then $e=e_{1}+\cdots+e_{n}, e^{\prime}=e_{n+1} \in C(E)$ and $x=x \wedge e+x \wedge e_{n+1}=$ $x \wedge e_{1}+\cdots+x \wedge e_{n}+x \wedge e_{n+1}$.

Let $e \in C(E)$, then the mapping $p_{e}: E \rightarrow[0, e]$ defined by

$$
p_{e}(x):=x \wedge e, \quad x \in E,
$$

is a homomorphism from $E$ onto $[0, e]$ whose kernel is $\left[0, e^{\prime}\right]$.

Proposition 2.6. Let $e, f \in C(E)$.

(i) $p_{e \wedge f}=p_{e} p_{f}=p_{f} p_{e}$.

(ii) If $e \wedge f=0$, then $e+f=e \vee f=f+e$ and $p_{e \vee f}(x)=p_{e}(x)+p_{f}(x)=$ $p_{f}(x)+p_{e}(x), x \in E$.

(iii) If $f \leq e$, then $e \backslash f=f \wedge e^{\prime}=f / e$, and $p_{e \wedge f^{\prime}}(x)=p_{e}(x) \backslash p_{f}(x)=$ $p_{f}(x) / p_{e}(x), x \in E$.

PROOF. (i) follows from (4).

(ii) If $e \wedge f=0$, then by Proposition 2.4, $e+f \in E$ and $f+e \in E$, and $e+f \geq e \vee f \leq f+e$. Hence $p_{e \vee f}(e+f)=p_{e \vee f}(e)+p_{e \vee f}(f)=e+f \leq e \vee f$. In an analogous way $f+e \leq e \vee f$.

Let $x \in E$. Then $p_{e \vee f}(x)=x \wedge(e \vee f)$ and $p_{e^{\prime} \wedge f^{\prime}}(x)=x \wedge e^{\prime} \wedge f^{\prime}$. Since $x=x \wedge e \wedge f+x \wedge e \wedge f^{\prime}+x \wedge e^{\prime} \wedge f+x \wedge e^{\prime} \wedge f^{\prime}=x \wedge e+x \wedge f+p_{(e \vee f)^{\prime}}(x)$, so that $p_{e \vee f}(x)=x \wedge e+x \wedge f$. If now $z \geq x \wedge e, x \wedge f$, then $z=z \wedge e \wedge f+z \wedge$ $e \wedge f^{\prime}+z \wedge e^{\prime} \wedge f+z \wedge e^{\prime} \wedge f^{\prime} \geq z \wedge e+z \wedge f \geq x \wedge e+x \wedge f$, which proves

$$
x \wedge(e \vee f)=(x \wedge e)+(x \wedge f)=(x \wedge e) \vee(x \wedge f)
$$

(iii) Let $f \leq e$. Then $e=f+f / e=e \backslash f+f$ and $e=e \wedge f+e \wedge f^{\prime}=$ $e \wedge f^{\prime}+e \wedge f$ so that $e \wedge f^{\prime}=f / e=e \backslash f=e \wedge f^{\prime}$. 
PROPOSITION 2.7. Let $e_{1}, \ldots, e_{n} \in C(E), e_{i} \wedge e_{j}=0$ for $i \neq j$.

(i) $e:=\bigvee_{i=1}^{n} e_{i}=e_{1}+\cdots+e_{n} \in C(E)$, and

$$
x \wedge e=\bigvee_{i=1}^{n}\left(x \wedge e_{i}\right)=x \wedge e_{1}+\cdots+x \wedge e_{n}, \quad x \in E .
$$

(ii) If $x_{i} \leq e_{i}$ for $i=1, \ldots, n$, then $x_{1}+\cdots+x_{n}=x_{1} \vee \cdots \vee x_{n}=x_{i_{1}}+\cdots+x_{i_{n}}$, where $\left(i_{1}, \ldots, i_{n}\right)$ is any permutation of $(1, \ldots, n)$.

(iii) If $a_{1}, \ldots, a_{n} \in C(E)$, then

$$
x \wedge\left(\bigvee_{i=1}^{n} a_{i}\right)=\bigvee_{i=1}^{n}\left(x \wedge a_{i}\right), \quad x \in E .
$$

PROOF. (i) If $n=1,2$, the assertion follows from Proposition 2.5 (ii). Let now the statement is true for any integer $i \leq n, n \geq 2$. Then $e=\bigvee_{i=1}^{n} e_{i} \vee e_{n+1}=$ $\left(e_{1}+\cdots+e_{n}\right)+e_{n+1}$ because $\left(\bigvee_{i=1}^{n} e_{i}\right) \wedge e_{n+1}=0$ due to the induction assumption. Hence $x \wedge e=x \wedge\left(\bigvee_{i=1}^{n} e_{i}\right) \vee x \wedge e_{n+1}=x \wedge e_{1}+\cdots+x \wedge e_{n}+x \wedge e_{n+1}$.

(ii) Since $e=e_{1}+\cdots+e_{n} \in E$, then $x=x_{1}+\cdots+x_{n} \in E$, and $x \geq x_{i}$ for any $i$. Assume $z \geq x_{i}$ for $i=1, \ldots, n$. Then by (ii) of Proposition 2.6 $z \geq p_{e}(z)=p_{e_{1}}(z)+\cdots+p_{e_{n}}(z) \geq p_{e_{1}}\left(x_{1}\right)+\cdots+p_{e_{n}}\left(x_{n}\right)=x_{1}+\cdots+x_{n}=x$. Consequently, $x=x_{1} \vee \cdots \vee x_{n}=x_{i_{1}}+\cdots+x_{i_{n}}$.

(iii) It is sufficient to assume $n=2$. Then define $e_{1}=a_{1} \wedge a_{2}^{\prime}, e_{2}=a_{1} \wedge a_{2}$, and $e_{3}=a_{1}^{\prime} \wedge a_{2}$. Hence by (i)

$$
\begin{aligned}
x \wedge\left(a_{1} \vee a_{2}\right) & =x \wedge\left(e_{1} \vee e_{2} \vee e_{3}\right)=\left(x \wedge e_{1}\right) \vee\left(x \wedge e_{2}\right) \vee\left(x \wedge e_{3}\right) \\
& =\left(\left(x \wedge e_{1}\right) \vee\left(x \wedge e_{2}\right)\right) \vee\left(\left(x \wedge e_{2}\right) \vee\left(x \wedge e_{3}\right)\right) \\
& =x \wedge\left(e_{1} \vee e_{2}\right) \vee x \wedge\left(e_{2} \vee e_{3}\right)=\left(x \wedge a_{1}\right) \vee\left(x \wedge a_{2}\right) .
\end{aligned}
$$

Proposition 2.8. Let $e \in C(E)$ and $f \leq e$. Then $f \in C(E)$ if and only if $f \in C([0, e])$.

PROOF. Let $f \in C(E)$. Then $e=f+e \wedge f^{\prime}$ and the product $[0, f] \times\left[0, e \wedge f^{\prime}\right]$ is isomorphic with $[0, e]$ under the mapping $f_{f}^{e}(x):=\left(x \wedge f, x \wedge f^{\sim^{e}}\right), x \in[0, e]$, so that $f \in C([0, e])$.

Conversely, let $f \in C([0, e])$. Then $E \cong[0, e] \times\left[0, e^{\sim}\right]$ and $[0, e] \cong[0, f] \times$ $\left[0, f^{\sim} \mathrm{c}\right]$. Since $1=e+e^{\sim}=f+f^{\sim e}+e^{\sim}$, then $f^{\sim}=f^{\sim e}+e^{\sim}=e \wedge f^{\sim} \cdot+e^{\sim}$. On the other hand, $f^{\sim}=f^{\sim} \wedge e+f^{\sim} \wedge e^{\sim}=f^{\sim} \wedge e+e^{\sim}$ while $e \in C(E)$ so that $f^{\sim} \wedge e=f^{\sim} \cdot \wedge e=e \backslash f=f^{\sim}$.

Take $x \in E$. Then $x \wedge f^{\sim}=x \wedge f^{\sim} \wedge e+x \wedge f^{\sim} \wedge e^{\sim}=x \wedge f^{\sim e}+x \wedge e^{\sim}$. Hence the mapping $\phi: E \rightarrow[0, f] \times\left[0, f^{\sim}\right]$ defined by $\phi(x):=\left(x \wedge f, x \wedge f^{\sim}\right), x \in E$, is an isomorphism in question while $E \cong[0, e] \times\left[0, e^{\sim}\right]$ and $x=x \wedge e+x \wedge e^{\sim}=$ $x \wedge e \wedge f+x \wedge e \wedge f^{\sim} \cdot+x \wedge e^{\sim}=x \wedge f+x \wedge f^{\sim e}+x \wedge e^{\sim}=x \wedge f+x \wedge f^{\sim}$. 
We note that in the case that $E$ is a quantum logic, for definitions see, for example, [8], then the centre of $E$ coincides with the set of all compatible elements of $E$.

It is worth to recall that the notion of a central element can be defined also for unital po-groups. We say that an element $e \in G$ of a unital po-group $(G, u)$ is central if (i) $0 \leq e \leq u$ and (ii) $e$ is a central element in the pseudo-effect algebra $\Gamma(G, u)=[0, u]$. In the case that $(G, u)$ is Abelian and with the Riesz interpolation property, then central elements coincide with characteristic elements [14, page 129].

A pseudo-effect algebra $E$ is said to be directly indecomposable if $E$ is non-trivial and whenever $E \cong E_{1} \times E_{2}$, then either $E_{1}$ or $E_{2}$ is trivial.

PROPOSITION 2.9. A pseudo-effect algebra $E$ is directly indecomposable if and only if $C(E)=\{0,1\}$.

Proof. Assume $E$ is directly indecomposable and let $e \in C(E)$. Then $E \cong$ $[0, e] \times\left[0, e^{\prime}\right]$ which means $e \in\{0,1\}$.

Conversely, let $C(E)=\{0,1\}$. The elements $(1,0)$ and $(0,1)$ are central elements in $E_{1} \times E_{2}$. If now $E \cong E_{1} \times E_{2}$ and if $\phi$ is an isomorphism from $E$ onto $E_{1} \times E_{2}$, set $e=\phi^{-1}((1,0))$. Then $e^{\sim}=e^{-}=\phi^{-1}((0,1))$, and $x \wedge e, x \wedge e^{\prime} \in E$ for any $x \in E$. Hence $\phi(x)=\phi(x) \wedge \phi(e)+\phi(x) \wedge \phi(e)^{\prime}=\phi\left((x \wedge e)+\left(x \wedge e^{\prime}\right)\right)$ which proves $x=x \wedge e+x \wedge e^{\prime}$. Hence $f_{e}(x):=\left(x \wedge e, x \wedge e^{\prime}\right)$ is an isomorphism of $E$ onto $[0, e] \times\left[0, e^{\prime}\right]$, so that, $e \in C(E)$. Therefore $e \in\{0,1\}$ and $\phi(e) \in\{0,1\}$ which proves $E$ is directly indecomposable.

We recall that a poset $E$ is an antilattice if an infimum of two elements exists only for comparable elements. Each linearly ordered poset is antilattice.

COROLlaRY 2.10. Every linearly ordered or antilattice pseudo-effect algebra is directly indecomposable.

Proof. It follows from Proposition 2.9, while in view of $0=e \wedge e^{\prime} \in\left\{e, e^{\prime}\right\}$, the centrum of a linearly ordered pseudo-effect algebra or of an antilattice pseudo-effect algebra is trivial, that is, $C(E)=\{0,1\}$.

\section{Pseudo-effect algebras and Riesz decomposition properties}

When we move from (commutative) effect algebras to pseudo-effect algebras, then the notion of the Riesz decomposition property can be extended to different and non-equivalent forms. Following [9], we introduce for pseudo-effect algebras the following forms of the Riesz decomposition properties: 
(a) For $a, b \in E$, we write $a \operatorname{com} b$ to mean that for all $a_{1} \leq a$ and $b_{1} \leq b, a_{1}$ and $b_{1}$ commute.

(b) We say that $E$ fulfills the Riesz interpolation property, (RIP) for short, if for any $a_{1}, a_{2}, b_{1}, b_{2} \in E$ such that $a_{1}, a_{2} \leq b_{1}, b_{2}$ there is a $c \in E$ such that $a_{1}, a_{2} \leq c \leq$ $b_{1}, b_{2}$.

(c) We say that $E$ fulfills the weak Riesz decomposition property, $\left(\mathrm{RDP}_{0}\right)$ for short, if for any $a, b_{1}, b_{2} \in E$ such that $a \leq b_{1}+b_{2}$ there are $d_{1}, d_{2} \in E$ such that $d_{1} \leq b_{1}$, $d_{2} \leq b_{2}$ and $a=d_{1}+d_{2}$.

(d) We say that $E$ fulfills the Riesz decomposition property, (RDP) for short, if for any $a_{1}, a_{2}, b_{1}, b_{2} \in E$ such that $a_{1}+a_{2}=b_{1}+b_{2}$ there are $d_{1}, d_{2}, d_{3}, d_{4} \in E$ such that $d_{1}+d_{2}=a_{1}, d_{3}+d_{4}=a_{2}, d_{1}+d_{3}=b_{1}, d_{2}+d_{4}=b_{2}$.

(e) We say that $E$ fulfills the commutational Riesz decomposition property, $\left(\mathrm{RDP}_{1}\right)$ for short, if for any $a_{1}, a_{2}, b_{1}, b_{2} \in E$ such that $a_{1}+a_{2}=b_{1}+b_{2}$ there are $d_{1}, d_{2}, d_{3}, d_{4} \in$ $E$ such that (i) $d_{1}+d_{2}=a_{1}, d_{3}+d_{4}=a_{2}, d_{1}+d_{3}=b_{1}, d_{2}+d_{4}=b_{2}$, and (ii) $d_{2} \operatorname{com} d_{3}$.

(f) We say that $E$ fulfills the strong Riesz decomposition property, $\left(\mathrm{RDP}_{2}\right)$ for short, if for any $a_{1}, a_{2}, b_{1}, b_{2} \in E$ such that $a_{1}+a_{2}=b_{1}+b_{2}$ there are $d_{1}, d_{2}, d_{3}, d_{4} \in E$ such that (i) $d_{1}+d_{2}=a_{1}, d_{3}+d_{4}=a_{2}, d_{1}+d_{3}=b_{1}, d_{2}+d_{4}=b_{2}$, and (ii) $d_{2} \wedge d_{3}=0$.

We have the implications

$$
\left(\mathrm{RDP}_{2}\right) \Rightarrow\left(\mathrm{RDP}_{1}\right) \Rightarrow(\mathrm{RDP}) \Rightarrow\left(\mathrm{RDP}_{0}\right) \Rightarrow(\mathrm{RIP}) \text {. }
$$

The converse of any of these implications does not hold. For commutative effect algebras we have

$$
\left(\mathrm{RDP}_{2}\right) \Rightarrow\left(\mathrm{RDP}_{1}\right) \Leftrightarrow(\mathrm{RDP}) \Leftrightarrow\left(\mathrm{RDP}_{0}\right) \Rightarrow(\mathrm{RIP}) .
$$

The following result was proved in [9, Lemma 3.2].

LEMMA 3.1. If $E$ satisfies $\left(\mathrm{RDP}_{0}\right)$, then $a \wedge b=0$ implies $a+b, b+a$ and $a \vee b$ exist in $E$ and are all equal.

THEOREM 3.2. Let a pseudo-effect algebra $E$ satisfy (RDP). Then $e \in E$ is central if and only if $e \wedge e^{\sim}=0$ if and only if $e \wedge e^{-}=0$.

Proof. Let $e \in C(E)$, then $e \wedge e^{\sim}=0=e \wedge e^{-}$. In view of Proposition 2.2 (iii) and $(\mathrm{v}), e \wedge e^{\sim}=0$ if and only if $e \wedge e^{-}=0$.

Conversely, let $e \wedge e^{\sim}=0$. Then $x \leq 1=e+e^{\sim}$ for any $x \in E$. There are $x_{1} \leq e$ and $x_{2} \leq e^{\sim}$ such that $x=x_{1}+x_{2}$. We show that if $y_{1} \leq e$ and $y_{2} \leq e^{\sim}$ and $x=y_{1}+y_{2}$, then $x_{1}=y_{1}$ and $x_{2}=y_{2}$. Due to (RDP), there are four elements $c_{11}, c_{12}, c_{21}, c_{22} \in E$ such that $x_{1}=c_{11}+c_{12}, x_{2}=c_{21}+c_{22}, y_{1}=c_{11}+c_{21}$ and $y_{2}=c_{12}+c_{22}$. Since $c_{12} \leq x_{1} \leq e$ and $c_{12} \leq y_{2} \leq e^{\sim}$, we conclude $c_{12}=0$. Similarly, $c_{21}=0$. Hence $x_{1}=c_{11}=y_{1}$ and $x_{2}=c_{22}=y_{2}$. 
Define $p_{e}(x)=x_{1}$ if $x=x_{1}+x_{2}(x \in E)$. Then $p_{e}: E \rightarrow[0, e]$. If $x_{1} \in[0, e]$ and $x_{2} \in\left[0, e^{\sim}\right]$, then $x_{1} \wedge x_{2}=0$, so that by Lemma 3.1, $x=x_{1}+x_{2}=x_{2}+x_{1}=x_{1} \vee x_{2}$, and hence $p_{e}(x)=x_{1}$. Consequently, $p_{e}$ restricted to $[0, e]$ is the identity.

We show that $p_{e}$ is a homomorphism. Let $x+y \in E$ and $x=x_{1}+x_{2}$ and $y=y_{1}+y_{2}$, where $x_{1}, y_{1} \leq e, x_{2}, y_{2} \leq e^{\sim}$. Then $x+y=x_{1}+x_{2}+y_{1}+y_{2}$. Since $x_{2} \wedge y_{1}=0$, then $x+y=x_{1}+y_{1}+x_{2}+y_{2}$. On the other hand, let $x+y=z_{1}+z_{2}$, where $z_{1} \leq e$ and $z_{2} \leq e^{\sim}$. Hence there are four elements $d_{11}, d_{12}, d_{21}, d_{22}$ such that

$$
\begin{array}{ll}
x_{1}+y_{1}=d_{11}+d_{12}, & z_{1}=d_{11}+d_{21}, \\
x_{2}+y_{2}=d_{21}+d_{22}, & z_{2}=d_{12}+d_{22} .
\end{array}
$$

We claim $d_{12}=0$. Since $d_{12} \leq x_{1}+y_{1}$, then $d_{12}=d^{\prime}+d^{\prime \prime}$, where $d^{\prime} \leq x_{1}$ and $d^{\prime \prime} \leq y_{1}$. Then $d^{\prime} \leq x_{1} \leq e$ and $d^{\prime} \leq d_{12} \leq z_{2} \leq e^{\sim}$ so that $d^{\prime}=0$, and $d^{\prime \prime} \leq y_{1} \leq e$ and $d^{\prime \prime} \leq z_{2} \leq e^{\sim}$ proving $d^{\prime \prime}=0$ and therefore $d_{12}=0$. In a similar way we can prove $d_{21}=0$ which yields $x_{1}+y_{1}=z_{1}$ and $x_{2}+y_{2}=z_{2}$, so that, $p_{e}$ is a homomorphism.

Since by Proposition $2.2(\mathrm{v}), e^{\sim}=e^{-}$, we can write $e^{\prime}:=e^{\sim}=e^{-}$, and let $p_{e^{\prime}}(x)=x_{2}$ if $x=x_{1}+x_{2}(x \in E)$. Then $p_{e^{\prime}}$ is a homomorphism from $E$ onto [0, $\left.e^{\prime}\right]$.

Consequently, the mapping $f_{e}: E \rightarrow[0, e] \times\left[0, e^{\prime}\right]$ defined by

$$
f_{e}(x)=\left(p_{e}(x), p_{e^{\prime}}(x)\right), \quad x \in E
$$

is an isomorphism with $f_{e}(e)=(e, 0)$, so that $e \in C(E)$. In addition,

$$
p_{e}(x)=x_{1}=x \wedge e, \quad x \in E
$$

\section{General comparability}

We say that a pseudo-effect algebra $E$ satisfies general comparability if, given $x, y \in E$, there is a central element $e \in E$ such that $p_{e}(x) \leq p_{e}(y)$ and $p_{e^{\prime}}(x) \geq$ $p_{\ell^{\prime}}(y)$. This means that the coordinates of the elements $x=\left(p_{e}(x), p_{\ell}(x)\right)$ and $y=\left(p_{e}(y), p_{e^{\prime}}(y)\right)$ can be compared in $[0, e]$ and $\left[0, e^{\prime}\right]$, respectively.

For example, (i) every linearly ordered pseudo-effect algebra trivially satisfies general comparability; (ii) also any Cartesian product of linearly ordered pseudoeffect algebras. For an 'MV-analogue' of the next result see [2, Proposition 3].

PROPOSITION 4.1. Let $M$ be a $\sigma$-complete pseudo $M V$-algebra and $x \in M$. Then the element $e:=\bigvee_{n=1}^{\infty}\left(x_{1} \oplus \cdots \oplus x_{n}\right)$, where $x_{n}=x$ for every $n$, is a central element of $M$ such that $p_{e}(x)=x$. If $f$ is any central element of $M$ such that $p_{f}(x)=x$, then $e \leq f$. Moreover, $M$ satisfies general comparability. 
ProOF. Let $M$ be a $\sigma$-complete pseudo MV-algebra. According to [7, Theorem 4.2], $M$ is commutative, that is, an MV-algebra. Let + be its partial addition defined via $a+b=a \oplus b$ if and only if $a \leq b^{*}$. Let $(G, u)$ be the unital $\ell$-group such that $M \cong \Gamma(G, u)$; such a group is guaranteed by Mundici's representation of MValgebras, see [20]. Then $G$ is Dedekind complete, and by [14, Lemma 9.8], the element $e=\bigvee_{i=1}^{\infty}(n x \wedge u) \in C(E)$ (compare with Theorem 3.2), and $p_{e}(x)=x$. Moreover, if $p_{f}(x)=x$ for some $f \in C(E)$, then $e \leq f$. Applying now [14, Theorem 9.9], $(G, u)$ satisfies general comparability, so $M$ satisfies general comparability.

THEOREM 4.2. Let $E$ be a pseudo-effect algebra satisfying general comparability. Then $E$ is a lattice, and $E$ can be organized into a pseudo $M V$-algebra such that the partial addition derived from $E$ as the pseudo $M V$-algebra coincides with the original + taken in the pseudo-effect algebra.

ProOF. Let $x, y \in E$ and let $e \in C(E)$ such that $p_{e}(x) \leq p_{e}(y)$ and $p_{e^{\prime}}(x) \geq$ $p_{e^{\prime}}(y)$. Then $x=p_{e}(x)+p_{e^{\prime}}(x) \geq p_{e}(x)+p_{e^{\prime}}(y)=: v \in E$.

Claim 1. $v=x \wedge y$.

PROOF. We have $y=p_{e}(y)+p_{e^{\prime}}(y) \geq p_{e}(x)+p_{e}(y)=v$, that is, $v \leq x, y$. Let $z \leq x, y$. Then $p_{e}(z) \leq p_{e}(x)$ and $p_{e^{\prime}}(z) \leq p_{e^{\prime}}(y)$, that is, $z=p_{e}(z)+p_{e^{\prime}}(z) \leq$ $p_{e}(x)+p_{e}(y)=v$, that is, $v=x \wedge y$.

Claim 2. $w:=p_{e}(y)+p_{e^{\prime}}(x) \in E$ and $w=x \vee y$.

PROOF. Since $p_{e}(y) \wedge p_{e^{\prime}}(x)=0$, then $w:=p_{e}(y)+p_{e^{\prime}}(x) \in E$. We conclude now $x \vee y=w$. We have $x=p_{e}(x)+p_{e^{\prime}}(x) \leq p_{e}(y)+p_{e^{\prime}}(x)=w$ and $y=$ $p_{e}(y)+p_{e^{\prime}}(y) \leq p_{e}(y)+p_{e^{\prime}}(x)=w$. If now $z \geq x, y$, then $p_{e}(z) \geq p_{e}(y)$ and $p_{e^{\prime}}(z) \geq p_{e^{\prime}}(x)$ that is, $z=p_{e}(z)+p_{e^{\prime}}(z) \geq w$.

Claim 3. $x \backslash(x \wedge y)=(x \vee y) \backslash y$ and $y \backslash(x \wedge y)=(x \vee y) \backslash x$.

Proof. Calculate

$$
\begin{aligned}
& p_{e}(x \backslash(x \wedge y))=p_{e}\left(x \backslash\left(p_{e}(x)+p_{e^{\prime}}(y)\right)=p_{e}(x) \backslash p_{e}(x)=0,\right. \\
& p_{e^{\prime}}(x \backslash(x \wedge y))=p_{e^{\prime}}(x) \backslash p_{e^{\prime}}(y), \quad p_{e}(y \backslash(x \wedge y))=p_{e}(y) \backslash p_{e}(x), \\
& p_{e^{\prime}}(y \backslash(x \wedge y))=p_{e^{\prime}}(y) \backslash p_{e^{\prime}}(y)=0, \\
& p_{e}((x \vee y) \backslash x)=p_{e}\left(\left(p_{e}(y)+p_{e^{\prime}}(x)\right) \backslash x\right)=p_{e}(y) \backslash p_{e}(x), \\
& p_{e^{\prime}}((x \vee y) \backslash x)=p_{e^{\prime}}(x) \backslash p_{e^{\prime}}(x)=0, \\
& p_{e}((x \vee y) \backslash y)=p_{e}(y) \backslash p_{e}(y)=0, \\
& p_{e^{\prime}}((x \vee y) \backslash y)=p_{e^{\prime}}(x) \backslash p_{e^{\prime}}(y),
\end{aligned}
$$

which proves Claim 3. 
Finally, according to [10, Proposition 8.7], Claim 3 is a necessary and sufficient condition in order to convert $E$ into a pseudo MV-algebra $\left(E ; \oplus,^{-}, \sim, 0,1\right)$; we define

$$
a \oplus b:=\left(a^{\sim} \backslash\left(a^{\sim} \wedge b\right)\right)^{-}, \quad a, b \in E .
$$

In such the case, the original + and the derived one from $\oplus$ coincide.

\section{Monotone $\sigma$-complete pseudo-effect algebras}

We say that a pseudo-effect algebra $E$ (i) is monotone $\sigma$-complete if any sequence $x_{1} \leq x_{2} \leq \cdots$ in $E$ has a supremum $\bigvee_{n=1}^{\infty} x_{n}$ in $E$; (ii) is $\sigma$-complete if $E$ is a $\sigma$-complete lattice; (iii) satisfies the countable Riesz interpolation property, ( $\sigma$-RIP) in abbreviation if, for countable sequences $\left\{x_{1}, x_{2}, \ldots\right\}$ and $\left\{y_{1}, y_{2}, \ldots\right\}$ of elements of $E$ such that $x_{i} \leq y_{j}$ for all $i, j$, there exists an element $z \in E$ such that $x_{i} \leq z \leq y_{j}$ for all $i, j$; (iv) is Archimedean if $n x:=x+\cdots+x$ is defined in $E$ for any integer $n \geq 1$, then $x=0$.

It is evident that ( $\sigma$-RIP) implies (RIP), and $E$ is monotone $\sigma$-complete if and only if each nonincreasing sequence of elements in $E$ has an infimum. Moreover, if $E$ is a lattice, then $E$ is monotone $\sigma$-complete if and only if $E$ is $\sigma$-complete.

PROPOSITION 5.1. Let E be a pseudo-effect algebra with (RIP). Then E has ( $\sigma$-RIP) if and only if whenever

(a) $x_{1} \leq x_{2} \leq \cdots$ in $E$ and $y_{1}, y_{2} \in E$, or

(b) $x_{1}, x_{2} \in E$ and $y_{1} \geq y_{2} \geq \cdots$ in $E$, or

(c) $x_{1} \leq x_{2} \leq \cdots$ and $y_{1} \geq y_{2} \geq \cdots$ in $E$,

and $x_{i} \leq y_{j}$ for all $i, j$ there exists $z \in E$ such that $x_{i} \leq z \leq y_{j}$ for all $i, j$.

PROOF. It follows the same steps as that in [14, Lemma 16.2].

Proposition 5.2. Let $E$ be a pseudo-effect algebra. Assume also that $\bigvee_{i} a_{i}$ and $\left(\bigvee_{i} a_{i}\right)+x \in E$, then $\bigvee_{i}\left(a_{i}+x\right) \in E$ and $\left(\bigvee_{i} a_{i}\right)+x=\bigvee_{i}\left(a_{i}+x\right)$. If $\bigvee_{i} a_{i}$, $x+\left(\bigvee_{i} a_{i}\right) \in E$, then $\bigvee_{i}\left(x+a_{i}\right) \in E$ and $x+\left(\bigvee_{i} a_{i}\right)=\bigvee_{i}\left(x+a_{i}\right)$.

PROOF. We have $\left(\bigvee_{i} a_{i}\right)+x \geq a_{i}+x$ for each $i$. If now $z \geq a_{i}+x$ for all $i$, then $z \backslash x \geq a_{i}$, that is, $z \backslash x \geq \bigvee_{i} a_{i}$ and $z \geq\left(\bigvee_{i} a_{i}\right)+x$.

PROPOSITION 5.3. Let E be a monotone $\sigma$-complete pseudo-effect algebra. Then $E$ is Archimedean. If, in addition, $E$ satisfies (RIP), then $E$ has countable interpolation. 
PROOF. Assume $x_{n}:=n x=x+\cdots+x$ be defined in $E$ for any $n \geq 1$. Then $x_{n} \leq x_{n+1}$ and there exists $x_{0}:=\bigvee_{n=1}^{\infty} x_{n}$. Since $x_{n} \leq x^{-}$for every $n$, then $x_{0} \leq x^{-}$, so that $x_{0}+x \in E$. Hence by Proposition 5.2,

$$
x_{0}+x=\bigvee_{n=1}^{\infty}\left(x_{n}+x\right)=\bigvee_{n=2}^{\infty} x_{n}=x_{0}
$$

which proves $x=0$.

If now $E$ has (RIP), then by Proposition 5.1, $E$ has countable interpolation.

The notion of monotone $\sigma$-complete pseudo-effect algebras is important while there are even (commutative) effect algebras which are monotone $\sigma$-complete but not a lattice.

EXAMPLE 5.4. There exists a monotone $\sigma$-complete effect algebra which is not a lattice.

PRoOF. Let $X$ be an uncountable set and fix two distinct elements $a, b \in X$. Let $E$ be the set of all functions $f: X \rightarrow \mathbb{Z}$ such that $f(x)=(f(a)+f(b)) / 2$ for all but countably many $x \in X$ and $0 \leq f(x) \leq 2$ for any $x \in X$. Then $E$ is an effect algebra which is monotone $\sigma$-complete but not a lattice. For example, let $u$ be the function which is the constant function 1 and let $v$ be a mapping in $E$ such that $v(a)=0$ and $v(b)=2$ while $v(x)=1$ for all $x \in X \backslash\{a, b\}$. Then $u$ and $v$ have no infimum in $E$ (see [14, Example 16.1, Example 16.8]).

We say that two elements $a$ and $b$ of a pseudo-effect algebra $E$ are compatible, and write $a \leftrightarrow b$ if there are three elements $a_{1}, b_{1}, c \in E$ such that $a=a_{1}+c, b=b_{1}+c$, and $a_{1}+b_{1}+c=b_{1}+a_{1}+c, a_{1} \wedge b_{1}=0$.

Proposition 5.5. Let $e \in C(E)$ and $x \in E$. Then $x \leftrightarrow e$, and

(i) $x=x \wedge e^{\prime}+x \wedge e, e=e \wedge x^{-}+x \wedge e, e=e \wedge x+e \wedge x^{\sim}, x=e \wedge x+x \wedge e^{\prime}$.

(ii) $x \wedge e^{\prime}=x \backslash(x \wedge e)=(x \wedge e) / x, e \wedge x^{-}=e \backslash(e \wedge x), e \wedge x^{\sim}=(e \wedge x) / x$.

(iii) $x \vee e=x \wedge e^{\prime}+e \wedge x^{-}+x \wedge e=e \wedge x^{-}+x \wedge e^{\prime}+x \wedge e=x \wedge e^{\prime}+x^{-} \wedge e+x^{\sim} \wedge e$.

(iv) $(x \vee e) \backslash e=x \backslash(x \wedge e),(x \vee e) \backslash x=e \backslash(x \wedge e)$, and $e /(x \vee e)=(x \wedge e) / x$, $x /(x \vee e)=(x \wedge e) / x$.

PROOF. (i) $x=x \wedge e^{\prime}+x \wedge e$ by Proposition 2.2. On the other hand, $p_{e}(e)=$ $e=e \wedge\left(x+x^{\sim}\right)=p_{e}(x)+p_{e}\left(x^{\sim}\right)=x \wedge e+x^{\sim} \wedge e$ and $e=e \wedge\left(x^{-}+x\right)=$ $p_{e}\left(x^{-}+x\right)=p_{e}\left(x^{-}\right)+p_{e}(x)=e \wedge x^{-}+e \wedge x$.

(ii) $x \backslash(x \wedge e) \in E$. If $x \backslash(x \wedge e)=a$, then $x=a+(x \wedge e)$, but $x=x \wedge e^{\prime}+x \wedge e$ which gives $a=x \wedge e^{\prime}$. Similarly, $(x \wedge e) / x=x \wedge e^{\prime}$, and also for other two equalities in (ii). 
(iii) We have $e=e \wedge 1=e \wedge\left(x^{-}+x\right)=x^{-} \wedge e+x \wedge e$. Since $x \wedge e^{\prime} \leq e^{\prime}$, then $x \wedge e^{\prime}+e \in E$ and $x, e \leq x \wedge e^{\prime}+e$. Moreover, if $z \geq x \wedge e^{\prime}, e$, then $p_{e}(z) \geq e$ and $p_{e^{\prime}}(z) \geq p_{e^{\prime}}\left(x \wedge e^{\prime}\right)=x \wedge e^{\prime}$ which proves $\left(x \wedge e^{\prime}\right) \vee e=x \wedge e^{\prime}+e$. It is clear that $x \vee e \leq x \wedge e^{\prime}+x^{-} \wedge e+e \wedge x$. If now $y \geq x, e$, then $y \geq x \wedge e^{\prime}, e$ and $y \geq\left(x \wedge e^{\prime}\right) \vee e$ which proves $x \vee e=x \wedge e^{\prime}+x^{-} \wedge e+x \wedge e$.

On the other hand, $x^{-} \wedge e \leq x^{-}$, we have $x^{-} \wedge e+x \in E$. Then $x^{-} \wedge e+x \geq x$ and $x^{-} \wedge e+x=x^{-} \wedge e+x \wedge e+x \wedge e^{\prime}=e+x \wedge e^{\prime} \geq e$. Therefore, if $u \geq x, e$, then $p_{e}(u) \geq e$ and $p_{e^{\prime}}(u) \geq p_{e^{\prime}}(x)=x \wedge e^{\prime}$ which entails $u \geq x^{-} \wedge e+x$ that is, $x^{-} \wedge e+x \wedge e^{\prime}+x \wedge e=x \vee e$.

(iv) By (iii) $(x \vee e) \backslash e=x \wedge e^{\prime}=x \backslash(x \wedge e)=(x \wedge e) / x$ and $x /(x \vee e)=$ $x /\left(x+x^{\sim} \wedge e\right)=x^{\sim} \wedge e=(e \wedge x) / x$.

From (iii) we have $(x \vee e) \backslash x=x^{-} \wedge e=e \backslash(x \wedge e)$. In a similar way we can prove the last equalities in (iv).

From the above we have also $x \leftrightarrow e$.

We recall that according to Proposition 5.5, if $e$ is a central element of $E$, then $e \leftrightarrow x$ for any $x \in E$. The converse statement is not true, for example, in any MV-algebra $M$ every two elements are compatible, and $C(M)=M$ if and only if $M$ is a Boolean algebra, see Theorem 3.2.

PROPOSITION 5.6. Let a pseudo-effect algebra E have ( $\sigma$-RIP). Let $\bigvee_{i=1}^{\infty} e_{i} \in C(E)$ for $e_{i} \in C(E), i \geq 1$. Then

$$
x \wedge\left(\bigvee_{i=1}^{\infty} e_{i}\right)=\bigvee_{i=1}^{\infty}\left(x \wedge e_{i}\right), \quad x \in E .
$$

Proof. First we show that

$$
\bigwedge_{i}\left(x \backslash\left(x \wedge e_{i}\right)\right)=x \backslash\left(x \wedge\left(\bigvee_{i} e_{i}\right)\right)
$$

We have $x \backslash\left(x \wedge e_{i}\right) \geq x \backslash\left(x \wedge\left(\bigvee_{i} e_{i}\right)\right)$. Let now $d \leq x \backslash\left(x \wedge e_{i}\right)$ for each $i$. By Proposition 5.5,

$$
d \leq x \backslash\left(x \wedge e_{i}\right)=\left(x \vee e_{i}\right) \backslash x \leq\left(x \vee\left(\bigvee_{i} e_{i}\right)\right) \backslash x=x \backslash\left(x \wedge\left(\bigvee_{i} e_{i}\right)\right)
$$

which proves (6).

It is clear that $\left(\bigvee_{i} e_{i}\right) \wedge x \geq e_{i} \wedge x$ for each $i$. Let now $e_{i} \wedge x \leq z$ for each $i$. Then $e_{i} \wedge x \leq z, x$ for each $i$. Applying ( $\sigma$-RIP), there exists an element $z_{0} \in E$ such that $e_{i} \wedge x \leq z_{0} \leq z, x$. Hence $x \backslash z_{0} \leq x \backslash\left(e_{i} \wedge x\right)$. Thus $x \backslash z_{0} \leq x \backslash\left(x \wedge\left(\bigvee_{i} e_{i}\right)\right)$ which gives $x \wedge\left(\bigvee_{i} e_{i}\right) \leq z_{0} \leq z$ and consequently (5) is proved. 
Proposition 5.7. Let a pseudo-effect algebra $E$ have ( $\sigma-\mathrm{RIP})$. Let $e=\bigvee_{i=1}^{\infty} e_{i} \in E$ for $e_{i} \in C(E), i \geq 1$. Then, for every $x \in E, x \wedge\left(\bigvee_{i=1}^{\infty} e_{i}\right)=\bigvee_{i=1}^{\infty}\left(x \wedge e_{i}\right), x \vee e \in E$, and $x \leftrightarrow e$.

PROOF. We have $e_{i} \wedge x \leq x, e$ for all $i$. Let $x_{0} \in E$ be any element such that $e_{i} \wedge x \leq x_{0} \leq e, x$ for every $i$; such an element always exists due to ( $\sigma$-RIP).

Claim 1. $x=\left(x \backslash x_{0}\right)+x_{0}, e=\left(e \backslash x_{0}\right)+x_{0},\left(x \backslash x_{0}\right)+\left(e \backslash x_{0}\right)+x_{0} \in E$.

PRoof. Indeed, we have $e_{i} \leq\left(x \backslash\left(e_{i} \wedge x\right)\right)^{\sim} \leq\left(x \backslash x_{0}\right)^{\sim}$ so that $e \leq\left(x \backslash x_{0}\right)^{\sim}$ which gives $\left(x \backslash x_{0}\right)+e \in E$.

Similarly, $e_{i} \leq\left(x \backslash\left(e_{i} \wedge x\right)\right)^{-} \leq\left(x \backslash x_{0}\right)^{-}$so that $e \leq\left(x \backslash x_{0}\right)^{-}$which gives $e+\left(x \backslash x_{0}\right) \in E$.

It is evident that $\left(x \backslash x_{0}\right)+e \geq x, e$ and $e+\left(x \backslash x_{0}\right) \geq x, e$.

Claim 2. $\bigwedge_{i}\left(x \backslash\left(x \wedge e_{i}\right)\right)=x \backslash x_{0}$.

PROOF. It is evident that $x \backslash\left(x \wedge e_{i}\right) \geq x \backslash x_{0}$ for every $i$. Let $d \leq x \backslash\left(x \wedge e_{i}\right)$ for each $i$. Then by Proposition 5.5, $d \leq x \backslash\left(x \wedge e_{i}\right)=\left(x \vee e_{i}\right) \backslash e_{i}$. Then $d+e_{i} \leq x \vee e_{i} \leq\left(x \backslash x_{0}\right)+e$ so that $e_{i} \leq d /\left(\left(x \backslash x_{0}\right)+e\right)$ and $e \leq d /\left(\left(x \backslash x_{0}\right)+e\right)$ which gives $d+e \leq\left(x \backslash x_{0}\right)+e$ and $d \leq x \backslash x_{0}$.

Claim 3. $\bigvee_{i}\left(x \wedge e_{i}\right)=x_{0}$

PROOF. Assume $x \wedge e_{i} \leq y$ for every $i$. Then $x \wedge e_{i} \leq y, x_{0}$ so that there exists $y_{0} \in E$ such that $x \wedge e_{i} \leq y_{0} \leq y, x_{0}$ for every $i$. Then $x \backslash y_{0} \leq x \backslash\left(x \wedge e_{i}\right)$. By Claim 2, $x \backslash y_{0} \leq \bigwedge_{i}\left(x \backslash\left(x \wedge e_{i}\right)\right)=x \backslash x_{0}$ so that $x_{0} \leq y_{0} \leq y$.

Claim 4. $\left(x \backslash x_{0}\right) \wedge\left(e \backslash x_{0}\right)=0$.

Proof. Assume $z \leq x \backslash x_{0}$ and $z \leq e \backslash x_{0}$. Then $z+x_{0} \leq x, z+x_{0} \leq e$, and $x \wedge e_{i} \leq z+x_{0} \leq e, x$ for each $i$. Using Claims 1-3, we have $z+x_{0}=\bigvee_{i}\left(x \wedge e_{i}\right)=x_{0}$, that is, $z=0$.

Claim 5. $x \wedge e=x_{0}$.

PROOF. Let $u \leq x, e$. Then $u, x_{0} \leq x, e$ and there exists $u_{0} \in E$ such that $u, x_{0} \leq u_{0} \leq x, e$, in particular, $x \wedge e_{i} \leq u_{0}$, and using Claims 1-3, we have $u_{0}=\bigvee_{i}\left(x \wedge e_{i}\right)=x_{0}$ that is, $u \leq u_{0}=x_{0}$ and $x_{0}=x \wedge e$.

Claim 6. $x_{0}+x \backslash x_{0} \in E, x_{0}+x \backslash x_{0}=x$ and $\left(e \backslash x_{0}\right)+\left(x \backslash x_{0}\right)+x_{0} \in E$. 
Proof. By Claim 1, $e+\left(x \backslash x_{0}\right) \in E$ so that $\left(e \backslash x_{0}\right)+\left(x_{0}+\left(x \backslash x_{0}\right)\right) \in E$ and $x_{0}+x \backslash x_{0} \in E$. Applying Proposition 5.2, we have

$$
x_{0}+x \backslash x_{0}=\bigvee_{i}\left(\left(x \wedge e_{i}\right)+\left(x \backslash x_{0}\right)\right)
$$

We show that, for each $i, x \wedge e_{i}+x \backslash x_{0}=x \backslash x_{0}+x \wedge e_{i}$. We recall that due to Claim 5 and Proposition 5.2, $x \backslash x_{0}+x \wedge e_{i} \in E$ for all $i$.

$$
\begin{aligned}
& p_{e_{i}}\left(x \wedge e_{i}+x \backslash x_{0}\right)=x \wedge e_{i}+\left(x \wedge e_{i}\right) \backslash\left(x_{0} \wedge e_{i}\right)=x \wedge e_{i}=p_{e_{i}}\left(x \backslash x_{0}+x \wedge e_{i}\right) \\
& p_{e_{i}}\left(x \wedge e_{i}+x \backslash x_{0}\right)=p_{e_{i}}\left(x \backslash x_{0}\right)=p_{e_{i}}\left(x \backslash x_{0}+x \wedge e_{i}\right)
\end{aligned}
$$

Applying again Proposition 5.2, we have for ( $\star$ )

$$
(\star)=\bigvee_{i}\left(x \backslash x_{0}+x \wedge e_{i}\right)=x \backslash x_{0}+\bigvee_{i}\left(x \wedge e_{i}\right)=x \backslash x_{0}+x_{0}=x
$$

Consequently, $e+x \backslash x_{0}=\left(e \backslash x_{0}\right)+x_{0}+\left(x \backslash x_{0}\right)=\left(e \backslash x_{0}\right)+\left(x \backslash x_{0}\right)+x_{0} \in E$.

Claim 7. $x \vee e=x \backslash x_{0}+e$.

PRoOF. We have $x, e \leq x \backslash x_{0}+e$ so that by (iii) of Proposition 5.5,

$$
x \vee e_{i}=x \wedge e_{i}^{\prime}+e_{i} \leq x \backslash x_{0}+e .
$$

Assume $x \vee e_{i} \leq v$ for all $i$. Then there exists $v_{0}$ such that $x \vee e_{i} \leq v_{0} \leq v, x \backslash x_{0}+e$. Then $x \vee e_{i}=x \backslash\left(x \wedge e_{i}\right)+e_{i} \geq\left(x \backslash x_{0}\right)+e_{i} \in E$. Since $x \backslash x_{0}+e \in E$, we can apply Proposition 5.2 and $\bigvee_{i}\left(\left(x \backslash x_{0}\right)+e_{i}\right)=x \backslash x_{0}+\bigvee_{i} e_{i}=x \backslash x_{0}+e \leq v_{0}$ which yields $v_{0}=x \backslash x_{0}+e \leq v$, that is, $x \backslash x_{0}+e=x \vee e$.

Claim 8. $\bigwedge_{i}\left(e \backslash\left(x \wedge e_{i}\right)\right)=e \backslash x_{0}$.

Proof. It is clear that $e \backslash\left(x \wedge e_{i}\right) \geq e \backslash x_{0}$. Assume $w \leq e \backslash\left(x \wedge e_{i}\right)$. There exists an element $w_{0} \in E$ such that $w, e \backslash x_{0} \leq w_{0} \leq \boldsymbol{e} \backslash\left(x \wedge \boldsymbol{e}_{i}\right)$ for each $i$. Hence $w_{0}+x \wedge e_{i} \leq e$ so that $x \wedge e_{i} \leq w_{0} / e$ and by Claim 5, $x_{0} \leq w_{0} / e$, that is, $w_{0}+x_{0} \leq e$ and $w_{0} \leq e \backslash x_{0}$.

Claim 9. $x \vee e=e \backslash x_{0}+x$.

PROof. By (iii) of Proposition 5.5, $x \vee e_{i}=e_{i} \wedge x^{-}+x=e_{i} \backslash\left(x \wedge e_{i}\right)+x \leq$ $e \backslash x_{0}+x$. Then $e_{i} \backslash\left(x \wedge e_{i}\right) \leq e \backslash x_{0} \leq e \backslash\left(x \wedge e_{i}\right)$ (Claim 8).

We now show that $\bigvee_{i}\left(e_{i} \backslash\left(x \wedge e_{i}\right)\right)=e \backslash x_{0}$. Assume $w \geq e_{i} \backslash\left(x \wedge e_{i}\right)$ for each $i$. Then there exists $w_{0} \in E$ such that $e_{i} \backslash\left(x \wedge e_{i}\right) \leq w_{0} \leq w, e \backslash x_{0}$. Therefore $\left(\boldsymbol{e} \backslash x_{0}\right) \backslash w_{0} \leq\left(e \backslash\left(x \wedge e_{i}\right)\right) \backslash\left(e_{i} \backslash\left(x \wedge e_{i}\right)\right)=e \backslash e_{i}$, that is, $\left(\left(\boldsymbol{e} \backslash x_{0}\right) \backslash w_{0}\right)+$ 
$\boldsymbol{e}_{i} \leq \boldsymbol{e}$ and $\boldsymbol{e}_{i} \leq\left(\left(\boldsymbol{e} \backslash x_{0}\right) \backslash w_{0}\right) / e$, and $\boldsymbol{e} \leq\left(\left(\boldsymbol{e} \backslash x_{0}\right) \backslash w_{0}\right) / e$. Consequently, $\left(\left(e \backslash x_{0}\right) \backslash w_{0}\right)+e \leq e$, that is, $e \backslash x_{0}=w_{0} \leq w$ which proves $e \backslash x_{0}=\bigvee_{i}\left(e_{i} \backslash(x \wedge\right.$ $\left.\left.e_{i}\right)\right)$. Applying Proposition 5.2, $e \backslash x_{0}+x=\bigvee_{i}\left(e_{i} \backslash\left(x \wedge e_{i}\right)+x\right)=\bigvee_{i}\left(e_{i} \vee x\right)=$ $e \vee x$.

Claim 10. $x \leftrightarrow e$.

It follows from the previous Claims.

PROPOSITION 5.8. Let E be a pseudo-effect algebra, $a=\bigvee_{i} a_{i} \in E$. Then

$$
\bigwedge_{i}\left(a \backslash a_{i}\right)=0=\bigwedge_{i}\left(a_{i} / a\right)
$$

PROOF. It is straightforward.

PROPOSITION 5.9. Let $E$ satisfy ( $\sigma$-RIP). If $a=\bigvee_{i=1}^{\infty} a_{i} \in E$ and $c \leq a_{i}$ for any $i$, then $\bigvee_{i}\left(a_{i} \backslash c\right), \bigvee_{i}\left(c / a_{i}\right) \in E$, and $a \backslash c=\bigvee_{i}\left(a_{i} \backslash c\right), c / a=\bigvee_{i}\left(c / a_{i}\right)$.

Proof. Since $c \leq a_{i} \leq a$, then $a_{i} \backslash c \leq a \backslash c$ for any $i$. Let $a_{i} \backslash c \leq v$ for any $i$. Then there exists an element $v_{0} \in E$ such that $a_{i} \backslash c \leq v_{0} \leq v, a \backslash c$. Hence, $(a \backslash c) \backslash v_{0} \leq(a \backslash c) \backslash\left(a_{i} \backslash c\right)=a \backslash a_{i}$. By Proposition 5.8, we have $(a \backslash c) \backslash v_{0}=0$, that is, $a \backslash c=v_{0} \leq v$, so that $a \backslash c=\bigvee_{i}\left(a_{i} \backslash c\right)$.

In a similar manner we can prove the second equality.

THEOREM 5.10. Let a pseudo-effect algebra E satisfy $(\sigma-\mathrm{RIP})$. Let $e=\bigvee_{i=1}^{\infty} e_{i} \in E$, where $e_{i} \in C(E), i \geq 1$. Then $e \in C(E)$.

PROOF. We recall that $e^{\sim}=e^{-}$. Indeed, $e^{\sim}=\bigwedge_{i} e_{i}^{\sim}=\bigwedge_{i} e_{i}^{-}$. Using Proposition 5.7, $e^{\prime} \wedge e=\bigvee_{i}\left(e^{\prime} \wedge e_{i}\right) \leq \bigvee_{i}\left(e_{i}^{\prime} \wedge e_{i}\right)=0$. Let $x \in E$ and $x_{0}=\bigvee_{i}\left(x \wedge e_{i}\right)$. Claim 1. $x \backslash(x \wedge e)=x \wedge e^{\sim}=e^{-} \wedge x=(x \wedge e) / x$.

PROOF. In view of Proposition 5.7, $x^{-} \vee e \in E$, and $\left(x^{-} \vee e\right)^{\sim}=x \wedge e^{\sim} \in E$. On the other hand, using Claim 2 of the proof of Proposition 5.7, we have

$$
x \backslash(x \wedge e)=x \backslash x_{0}=\bigwedge_{i}\left(x \backslash\left(x \wedge e_{i}\right)\right)=\bigwedge_{i}\left(x \wedge e_{i}^{\sim}\right)=x \wedge \bigwedge_{i} e_{i}^{\sim}=x \wedge e^{\sim}
$$

It is possible to show $(x \wedge e) / x=\wedge_{i}\left(\left(x \wedge e_{i}\right) / x\right)$. Define $p_{e}(x):=x \wedge e$ and $p_{e^{\prime}}(x):=x \wedge e^{\sim}$. Then $p_{e}(x)+p_{e^{\prime}}(x)=x=p_{e^{\prime}}(x)+p_{e}(x)$.

Claim 2. If $y \leq x$, then $(x \backslash y) \wedge e=(x \wedge e) \backslash(y \wedge e)$. 
PROOF. We have $(x \wedge y) \wedge e=\bigvee_{i}\left((x \backslash y) \wedge e_{i}\right)=\bigvee_{i}\left(\left(x \wedge e_{i}\right) \backslash\left(y \wedge e_{i}\right)\right) \geq$ $\left(x \wedge e_{i}\right) \backslash(y \wedge e)$. Applying Proposition 5.9, we have

$$
\begin{aligned}
\bigvee_{i}\left(\left(x \wedge e_{i}\right) \backslash(y \wedge e)\right) & =\left(\bigvee_{i}\left(x \wedge e_{i}\right) \backslash(y \wedge e)\right) \\
& =(x \wedge e) \backslash(y \wedge e) \leq(x \backslash y) \wedge e .
\end{aligned}
$$

On the other hand, $(x \backslash y) \wedge e=\bigvee_{i}\left(\left(x \wedge e_{i}\right) \backslash\left(y \wedge e_{i}\right)\right) \leq(x \wedge e) \backslash\left(y \wedge e_{i}\right)$. Assume $d \leq(x \wedge e) \backslash\left(y \wedge e_{i}\right)$ for each $i$. Then there exists $d_{0} \in E$ such that $d,(x \backslash y) \wedge e \leq d_{0} \leq(x \wedge e) \backslash\left(y \wedge e_{i}\right)$ for any $i$. Therefore, $d_{0}+\left(y \wedge e_{i}\right) \leq x \wedge e$, $y \wedge e_{i} \leq d_{0} /(x \wedge e)$, so that

$$
y \wedge e \leq d_{0} /(x \wedge e) \text { and } d_{0} \leq(x \wedge e) \backslash(y \wedge e) .
$$

Combining $(*)$ and $(* *)$, we have $(x \backslash e) \wedge e=(x \wedge e) \backslash(y \wedge e)$.

Claim 3. If $x+y \in E$, then $(x+y) \wedge e=x \wedge e+y \wedge e$.

Proof. Due to Claim 2, $x \wedge e=((x+y) \backslash y) \wedge e=((x+y) \wedge e) \backslash(y \wedge e)$.

Claim 4. If $x+y \in E$, then $(x+y) \wedge e^{\prime} \geq x \wedge e^{\prime}+y \wedge e^{\prime}$.

PROOF. $(x+y) \wedge e^{\prime}=\bigwedge_{i}\left((x+y) \wedge e_{i}^{\prime}\right)=\bigwedge_{i}\left(x \wedge e_{i}+y \wedge e_{i}\right) \geq x \wedge e^{\prime}+y \wedge e^{\prime}$.

Claim 5. If $x \leq e, y \leq e^{\prime}$, then $x+y=x \vee y=y+x$.

PROOF. Due to $e+e^{\prime}=1=e^{\prime}+e$, we have $x+y, y+x \in E$, and $x+y \geq x, y$. Assume $z \geq x, y$. There exists $z_{0} \in E$ such that $z, x+y \geq z_{0} \geq x+y$. Then $p_{e}\left(z_{0}\right) \geq x, p_{e^{\prime}}\left(z_{9}\right) \geq y$, that is, $z_{0}=p_{e}\left(z_{0}\right)+p_{e^{\prime}}\left(z_{0}\right) \geq x+y$, that is, $x+y=x \vee y$.

We have $x+y=p_{e}(x+y)+p_{e^{\prime}}(x+y)=p_{e^{\prime}}(x+y)+p_{e}(x+y) \geq y+x$. But $y+x \geq x, y$, then $y+x \geq x \vee y=x+y$.

Claim 6. If $x \leq e, y \leq e^{\prime}$, then $(x+y) \wedge e^{\prime}=x \wedge e^{\prime}+y \wedge e^{\prime}$.

Proof. Using Claim 5, we have

$$
\begin{aligned}
x+y & =p_{e}(x+y)+p_{e^{\prime}}(x+y) \geq p_{e}(x)+p_{e}(y)+p_{e^{\prime}}(x)+p_{e^{\prime}}(y) \\
& =p_{e}(x)+p_{e^{\prime}}(x)+p_{e}(y)+p_{e^{\prime}}(y)=x+y,
\end{aligned}
$$

which proves $p_{e^{\prime}}(x+y)=p_{e^{\prime}}(x)+p_{e^{\prime}}(y)$.

Claim 7. $E \cong[0, e] \times\left[0, e^{\sim}\right]$. 
Proof. Define $f_{e}(x):=\left(x \wedge e, x \wedge e^{\prime}\right), x \in E$. Then $x=x \wedge e+x \wedge e^{\prime}$ and if $f_{e}(x)=f_{e}(y)$, then $x=y$. According to Claim 3 and Claim $6, f_{e}$ is an injective homomorphism from $E$ into $[0, e] \times\left[0, e^{\sim}\right], f_{e}(e)=(e, 0)$ and due to Claim $5, f_{e}$ is surjective.

Summarizing all claims, we finally have $e \in C(E)$.

THEOREM 5.11. Let a pseudo-effect algebra $E$ be monotone $\sigma$-complete. Let $e=\bigvee_{i=1}^{\infty} e_{i} \in E$, where $e_{i} \in C(E), i \geq 1$. Then $e \in C(E)$, and

$$
x \wedge\left(\bigvee_{i=1}^{\infty} e_{i}\right)=\bigvee_{i=1}^{\infty}\left(x \wedge e_{i}\right), \quad x \in E .
$$

PROOF. Since by Theorem 2.3, $C(E)$ is a Boolean algebra, without loss of generality we can assume $e_{1} \leq e_{2} \leq \cdots$. Therefore, $e \in E$. In addition $x \wedge e_{i} \in E$, which entails $x_{0}:=\bigvee_{i}\left(x \wedge e_{i}\right)$ is defined in $E$, and $x_{0} \leq x, e$.

Using a slightly modified proof of Proposition 5.7, we can show that if $x_{0}^{*}$ is any element of $E$ such that $x \wedge e_{i} \leq x_{0}^{*} \leq x, e$ for any $i$, then $x_{0}=x_{0}^{*}$. In addition, Claim 1, Claim 2, Claim 4, Claim 6, and Claim 8 in the proof of Proposition 5.7 are also true, and $x \backslash x_{0}=\bigwedge_{i}\left(x \backslash\left(x \wedge e_{i}\right)\right)=x_{0} / x$, hence $x \backslash x_{0}+x_{0}=x=x_{0}+x_{0} / x$.

Claim 1. $e \wedge e^{\prime}=0$.

PRoOF. Assume $z \leq e, e^{\prime}$, then $z_{0}=\bigvee_{i}\left(z \wedge e_{i}\right) \leq z \leq e, e^{\prime}$. Therefore, $z \wedge e_{i} \leq e_{i}$ and $z \wedge e_{i} \leq z_{4} \leq e^{\prime} \leq e_{i}^{\prime}$ so that $z_{0}=0$. Then $z \backslash z_{0} \leq e \backslash z_{0}$ and by Claim 4 of Proposition 5.7, we have $z \backslash z_{0}=\left(z \backslash z_{0}\right) \wedge\left(e \backslash z_{0}\right)=0$, that is, $z=0$.

Define two mappings $q_{e}: E \rightarrow[0, e]$ and $q_{e^{\prime}}: E \rightarrow\left[0, e^{\prime}\right]$ by

$$
q_{e}(x):=\bigvee_{i}\left(x \wedge e_{i}\right)=: x_{0}, \quad q_{\ell^{\prime}}(x):=x \backslash x_{0}
$$

for any $x \in E$. Then $q_{e}(e)=e$ and $q_{e^{\prime}}(e)=0$.

Claim 2. If $x+y \in E$, then $q_{e}(x+y)=q_{e}(x)+q_{e}(y)$, and $q_{e}$ is monotone.

PROOF. Calculate, $q_{e}(x+y)=\bigvee_{i}\left((x+y) \wedge e_{i}\right)=\bigvee_{i}\left(x \wedge e_{i}+y \wedge e_{i}\right) \leq$ $q_{e}(x)+q_{e}(y) \in E$.

Assume $(x+y) \wedge e_{i} \leq z$ for any $i$, and fix an integer $i_{0} \geq 1$. Then $x_{0}, y_{6} \leq z$ and $x \wedge e_{i}+y \wedge e_{i_{0}} \leq z$ for any $i \geq i_{0}$. Hence $x \wedge e_{i} \leq z \backslash\left(y \wedge e_{i_{0}}\right)$, that is, $x_{0} \leq z \backslash\left(x \wedge e_{i_{0}}\right)$ and $y \wedge e_{i 9} \leq x_{0} / z$ which gives $y_{0} \leq x_{0} / z$ and $x_{0}+y_{0} \leq z$.

Claim 3. If $x+y \in E$, then $q_{e^{\prime}}(x+y) \geq q_{e^{\prime}}(x)+q_{e^{\prime}}(y)$, and $q_{e^{\prime}}$ is monotone.

Indeed, $q_{e^{\prime}}(x+y)=\bigwedge_{i}\left((x+y) \wedge e_{i}^{\prime}\right)=\bigwedge_{i}\left(x \wedge e_{i}^{\prime}+y \wedge e_{i}^{\prime}\right) \geq x \wedge e^{\prime}+y \wedge e^{\prime} \in E$. 
Claim 4. If $x \leq e, y \leq e^{\prime}$, then $q_{e}(x)=x$ and $q_{e^{\prime}}(y)=y$.

Calculate, $q_{e}(x)=x_{0}$ and $q_{e^{\prime}}(x)=x \backslash x_{0} \leq e, e^{\prime}$ which by Claim 1 means $x \backslash x_{0}=0$. Similarly we prove $q_{e^{\prime}}(y)=y$.

Claim 5. If $x \leq e$ and $y \leq e^{\prime}$, thon $x+y=x \vee y=y+x$.

Proof. Since $x \leq e$ and $y \leq e^{\prime}$, we have $x+y, y+x \in E$, and $x+y \geq x, y$. Assume $z \geq x, y$. Then $q_{e}(z) \geq q_{e}(x)=x$ and $q_{e}(z) \geq q_{e^{\prime}}(y)=y$ which gives $z=q_{e}(z)+q_{e^{\prime}}(z) \geq x+y$, that is, $x+y=x \vee y$.

We assert that $q_{e^{\prime}}(x+y)=y$. Indeed, $x+y=q_{e}(x+y)+q_{e^{\prime}}(x+y) \geq$ $q_{e}(x)+q_{e}(y)+q_{e^{\prime}}(x)+q_{e^{\prime}}(y)=x+y$.

Assume now $x+y=y+d$ for some $d \in E$. Then $x=q_{e}(x+y)=q_{e}(y+d)=$ $q_{e}(d)$ and $y=q_{e^{\prime}}(x+y)=q_{e^{\prime}}(y+d) \geq y+q_{e^{\prime}}(d)$ which implies $x+y=y+d \geq$ $y+q_{e}(d)=y+x$. But $y+x \geq x, y$, then $y+x \geq x \vee y=x+y$.

Claim 6. If $x+y \in E$, then $q_{e^{\prime}}(x+y)=q_{e^{\prime}}(x)+q_{e^{\prime}}(y)$.

Calculate and use Claim 5,

$$
\begin{aligned}
x+y & =q_{e}(x+y)+q_{e^{\prime}}(x+y) \geq q_{e}(x)+q_{e}(y)+q_{e^{\prime}}(x)+q_{e^{\prime}}(y) \\
& =q_{e}(x)+q_{e^{\prime}}(x)+q_{e}(y)+q_{e^{\prime}}(y)=x+y .
\end{aligned}
$$

Claim 7. If $f_{e}: E \rightarrow[0, e] \times\left[0, e^{\prime}\right]$ is defined by $f_{e}(x)=\left(q_{e}(x), q_{e^{\prime}}(x)\right), x \in E$, then $f_{e}$ is an isomorphism and $e \in C(E)$.

Indeed, $f_{e}(e)=(e, 0)$, and if $f_{e}(x)=\left(x_{1}, x_{2}\right)$, then $x=x_{5}+x_{2}$, and by Claim 2 and Claim 6, $f_{e}$ is an injective homomorphism. Assume $x \leq e$ and $y \leq e^{\prime}$, then $x+y \in E$ and $f_{e}(x+y)=(x, y)$, which proves that $e$ is a central element of $E$.

Therefore, $x \wedge e \in E$, so that $x \wedge e=x_{0}$, and in addition, $q_{e}=p_{e}$, where $p_{e}$ is defined by (4).

\section{The Cantor-Bernstein theorem for pseudo-effect algebras}

In the present section, we apply the notion of central elements to show that an analogue of the Cantor-Bernstein theorem for pseudo-effect algebras can be obtained. We will study the case when the centre of a pseudo-effect algebra $E$ is a Boolean $\sigma$-subalgebra of $E$ with the central decomposition property.

We say that a finite or countable sequence $\left\{e_{i}\right\}$ of central elements of a pseudoeffect algebra $E$ is a central decomposition of unity if $e_{i} \wedge e_{j}=0$ for any $i \neq j$, and $\bigvee_{i} z_{i}=1 . E$ is said to satisfy the central decomposition property if (i) any sequence of central elements $\left\{f_{i}\right\}$ has a supremum in $E$ and belongs to $C(E)$ and $x \wedge\left(\bigvee_{i} f_{i}\right)=\bigvee_{i}\left(x \wedge f_{i}\right)$ for any $x \in E$, and (ii) if $\left\{e_{i}\right\}$ is a central decomposition of unity and $x_{i} \leq e_{i}$ for any $i$, then $x=\bigvee_{i} x_{i} \in E$. That is if $\left\{e_{i}\right\}_{i}$ is a sequence of 
central elements of $E$, then $e=\bigvee_{i} e_{i} \in E$ and $e \in C(E)$. For example, any monotone $\sigma$-complete pseudo-effect algebra has such a property, see Proposition 6.1 below and Theorem 5.11, and every linearly ordered pseudo-effect algebra has the centrum $C(E)=\{0,1\}$ which is a Boolean $\sigma$-algebra but $E$ is not necessarily monotone $\sigma$ complete and it has this property as well as any Cartesian product of finitely many linearly ordered pseudo-effect algebras.

PROPOSITION 6.1. (i) Let $e_{1}, \ldots e_{n}$ be a finite central decomposition of unity in a pseudo-effect algebra $E$. The mapping $\phi: E \rightarrow \prod_{i=1}^{n}\left[0, e_{i}\right]$ given by $\phi(x)=$ $\left(x \wedge e_{i}\right)_{i}, x \in X$, is an isomorphism.

(ii) Let $\left\{e_{i}\right\}_{i}$ be a countable central decomposition of unity in a monotone $\sigma$ complete pseudo-effect algebra $E$. The mapping $\phi: E \rightarrow \prod_{i}\left[0, e_{i}\right]$ given by $\phi(x)=$ $\left(x \wedge e_{i}\right)_{i}, x \in E$, is an isomorphism, and $E$ satisfies the central decomposition property.

PROOF. (i) It follows from Proposition 2.7.

(ii) It is clear the mapping $\phi$ is an injective homomorphism. Assume now $x_{i} \leq e_{i}$ for any $i$. By (ii) of Proposition 2.7, $x^{n}=x_{1}+\cdots+x_{n} \in E$ for any $n \geq 1$. Then $x=\bigvee_{n} x^{n}=\bigvee_{i} x_{i} \in E$ and $\phi(x)=\left(x \wedge e_{i}\right)_{i}=\left(x_{i}\right)_{i}$ which proves that $\phi$ is surjective. The central decomposition property follows now from Theorem 5.11 .

PROPOSITION 6.2. Let $E$ and $F$ be two pseudo-effect algebras and let $f \in C(F)$. Assume that $h$ is an isomorphism from $E$ onto $[0, f]$. If $e \in C(E)$, then $h(e) \in C(F)$.

ProOF. In view of Proposition 2.8, it is sufficient to show that $h(e) \in C([0, f])$. Put $f_{0}:=h(e)$. Then $f_{0}^{-f}=f_{0}^{\sim f}$. For any $y \in[0, f], y \wedge f_{0}, y \wedge f_{0}^{\sim f} \in[0, f]$; indeed, we have $h(x)=y$ for a unique $x \in E$ and $x \wedge e \in E, x \wedge e^{\sim} \in E$, so that $h(x \wedge e)=h(x) \wedge h(e)=e \wedge f_{0}$ and $h\left(x \wedge e^{\sim}\right)=h(x) \wedge h\left(e^{\sim}\right)=y \wedge f_{0}^{\sim \prime}$. Therefore the mapping $\phi:[0, f] \rightarrow\left[0, f_{0}\right] \times\left[0, f_{0}^{\sim f}\right]$ defined by $\phi_{f}(y)=\left(y \wedge f_{0}, y \wedge f_{0}^{\sim f}\right)$, $y \in[0, f]$, is an isomorphism in question proving $f_{0} \in C([0, f])$.

THEOREM 6.3 (Cantor-Bernstein). Let $E$ and $F$ be pseudo-effect algebras satisfying the central decomposition property. Let $e \in C(E)$ and $f \in C(F)$ and let there are two isomorphisms of pseudo-effect algebras $\alpha: E \rightarrow[0, f]$ and $\beta: F \rightarrow[0, e]$. Then $E$ and $F$ are isomorphic pseudo-effect algebras.

PROOF. Without loss of generality we can assume that $0<e<1$ and $0<f<1$. Define recursively two sequences $\left\{e_{n}\right\}_{n=0}^{\infty}$ and $\left\{f_{n}\right\}_{n=0}^{\infty}$ by

$$
\begin{aligned}
e_{0} & =1, & e_{n+1} & =\beta\left(f_{n}\right), \\
f_{0} & =1, & f_{n+1} & =\alpha\left(e_{n}\right) .
\end{aligned}
$$


Due to Proposition 6.2, $e_{n} \in C(E)$ and $f_{n} \in C(F)$ for each $n \geq 0$. In addition, $e_{0} \geq e_{1} \geq e_{2} \geq \cdots$ in $E$ and $f_{0} \geq f_{1} \geq f_{2} \geq \cdots$ in $F$. By the assumptions, the elements $e_{\infty}=\bigwedge_{n=1}^{\infty} e_{n}$ and $f_{\infty}=\bigwedge_{n=0}^{\infty} f_{n}$ are defined in $E$ and $F$, respectively, and, in addition, $e_{\infty} \in C(E)$ and $f_{\infty} \in C(F)$. For all $n$ we have $e_{n+2}=(\beta \circ \alpha)\left(e_{n}\right)$ and $f_{n+2}=(\alpha \circ \beta)\left(f_{n}\right)$. The mapping $\beta \circ \alpha$ is an isomorphism of $E$ onto $\left[0, e_{2}\right]$, and it preserves countable infima and suprema. Therefore, $(\beta \circ \alpha)\left(e_{\infty}\right)=(\beta \circ \alpha)\left(\bigwedge_{n} e_{n}\right)=$ $\bigwedge_{n}(\beta \circ \alpha)\left(e_{n}\right)=\bigwedge_{n} e_{n+2}$. Analogously, $f_{\infty}=(\alpha \circ \beta)\left(f_{\infty}\right)$, and $e_{\infty}=0$ if and only if $f_{\infty}=0$ while $\alpha\left(e_{\infty}\right)=f_{\infty}$ and $\beta\left(f_{\infty}\right)=e_{\infty}$. It is evident that the sequences $\left(e_{\infty}, e_{0} \backslash e_{1}, e_{1} \backslash e_{2}, \ldots\right)$ and $\left(f_{\infty}, f_{0} \backslash f_{1}, f_{1} \backslash f_{2}, \ldots\right)$ are decompositions of unity in $E$ and $F$, respectively.

Moreover, if $x \in E$ and $y \in F$, then $[0, x]$ is isomorphic with $[0, \alpha(x)]$ and $[0, y]$ is isomorphic with $[0, \beta(y)]$. The restrictions of $\alpha$ and $\beta^{-1}$ induce isomorphisms

$$
\begin{gathered}
{\left[0, e_{\infty}\right] \cong\left[0, f_{\infty}\right], \quad\left[0, e_{2 n-2} \backslash e_{2 n-1}\right] \cong\left[0, f_{2 n-1} \backslash f_{2 n}\right],} \\
{\left[0, e_{2 n-1} \backslash e_{2 n}\right] \cong\left[0, f_{2 n-2} \backslash f_{2 n-1}\right] .}
\end{gathered}
$$

By assumptions,

$$
E \cong\left[0, e_{\infty}\right] \times \prod_{n=0}^{\infty}\left[0, e_{n} \backslash e_{n+1}\right] \text { and } F \cong\left[0, f_{\infty}\right] \times \prod_{n=0}^{\infty}\left[0, f_{n} \backslash f_{n+1}\right],
$$

consequently $E \cong F$.

REMARK 6.4. (1) Theorem 6.3 generalizes the result of [4] for $\sigma$-complete MValgebras.

(2) Theorem 6.3 generalizes the result of Jenča [18] for monotone $\sigma$-complete (commutative) effect algebras.

\section{Acknowledgement}

The author is very indebted to the referees for their valuable suggestions which improved the readability of the paper.

The paper has been supported by the grant VEGA 2/7193/20 SAV, Bratislava, Slovakia.

\section{References}

[1] R. Baudot, 'Non-commutative logic programming language NoClog', in: Symposium LICS (Santa Barbara, 2000, Short Presentation) pp. 3-9. 
[2] L. P. Belluce, ' $\alpha$-complete MV-algebras', in: Non-classical logics and their applications to fuzzy subsets (ed. U. Hoehle et al.) (Kluwer, Dordrecht, 1995) pp. 7-21.

[3] C. C. Chang, 'Algebraic analysis of many valued logics', Trans. Amer. Math. Soc. 88 (1958), $467-490$.

[4] A. De Simone, D. Mundici and M. Navara, 'A Cantor-Bernstein theorem for $\sigma$-complete MValgebras', Czechoslovak Math. J. (to appear).

[5] A. De Simone, M. Navara and P. Pták, 'On interval homogeneous orthomodular lattices', Comm. Math. Univ. Carolinae 42 (2001), 23-30.

[6] A. Di Nola, G. Georgescu and A. Iorgulescu, 'Pseudo-BL-algebras, I, II', Mult.-Valued Logic (to appear).

[7] A. Dvurečenskij, 'Pseudo MV-algebras are intervals in $\ell$-groups', J. Austral. Math. Soc. 72 (2002), 427-445.

[8] A. Dvure đenskij and S. Pulmannová, New trends in quantum structures (Kluwer, Dordrecht, 2000).

[9] A. Dvurečenskij and T. Vetterlein, 'Pseudoeffect algebras. I. Basic properties', Internat. J. Theor. Phys. 40 (2001), 685-701.

[10] - 'Pseudoeffect algebras. II. Group representations', Internat. J. Theor. Phys. 40 (2001), 703-726.

[11] D. J. Foulis and M. K. Bennett, 'Effect algebras and unsharp quantum logics', Found. Phys. 24 (1994), 1325-1346.

[12] G. Georgescu and A. Iorgulescu, 'Pseudo-MV algebras: A non-commutative extension of MValgebras', in: Proc. Fourth Inter. Symp. on Econ. Inform., May 6-9, 1999, Bucharest (ed. I. Smeureanu et al.) (INFOREC Printing House, Bucharest, 1999) pp. 961-968.

[13] — 'Pseudo-MV algebras', Mult.-Valued Logic 6 (2001), 95-135.

[14] K. R. Goodearl, Partially ordered abelian groups with interpolation, Math. Surveys and Monographs 20 (Amer. Math. Soc., Providence, RI, 1986).

[15] R. J. Greechie, D. J. Foulis and S. Pulmannová, 'The center of an effect algebra', Order 12 (1995), 91-106.

[16] J. Jakubík, 'Cantor-Bernstein theorem for MV-algebras', Czechoslovak Math. J. 49 (1999), 517529.

[17] - 'A theorem of Cantor-Bernstein theorem type for orthogonally $\sigma$-complete pseudo MValgebras', Tatra Mt. Math. Publ. (to appear).

[18] G. Jenča, 'A Cantor-Bernstein type theorem for effect algebras', Algebra Universalis (to appear).

[19] F. Kôpka and F. Chovanec, 'D-posets', Math. Slovaca 44 (1994), 21-34.

[20] D. Mundici, 'Interpretation of AF $C^{*}$-algebras in Łukasiewicz sentential calculus', J. Funct. Anal. 65 (1986), 15-63.

[21] J. Rachünek, 'A non-commutative generalization of MV-algebras', Czechoslovak Math. J. 52 (2002), 255-273.

Mathematical Institute

Slovak Academy of Sciences

Štefánikova 49

SK-814 73 Bratislava

Slovakia

e-mail: dvurecen@mat.savba.sk 
